\title{
Understanding contextual interactions to design navigational context-aware applications
}

\author{
Nicholas A. Bradley, Mark D. Dunlop \\ Department of Computer and Information Sciences \\ University of Strathclyde, Glasgow, Scotland, G1 1XH, UK \\ Tel: +44 (0) 141552 4400, Fax: +44 (0) 1415525330 \\ E-mail: \{Nick.Bradley, Mark.Dunlop\}@ cis.strath.ac.uk
}

\begin{abstract}
Context-aware technology has stimulated rigorous research into novel ways to support people in a wide range of tasks and situations. However, the effectiveness of these technologies will ultimately be dependent on the extent to which contextual interactions are understood and accounted for in their design. This study involved an investigation of contextual interactions required for route navigation. The purpose was to illustrate the heterogeneous nature of humans in interaction with their environmental context. Participants were interviewed to determine how each interacts with or use objects/information in the environment in which to navigate/orientate. Results revealed that people vary individually and collectively. Usability implications for the design of navigational context-aware applications are identified and discussed.
\end{abstract}

\section{Introduction}

Context-aware technology has stimulated a growth of research and development into novel ways in which to support the user in a wide range of tasks and situations. Context-aware applications are designed to discover and take advantage of contextual information such as a user's location, time of day, nearby people and devices, and user activity [1]. Examples include (i) context-aware mobile tourist guides [2] and (ii) location-aware shopping assistants [3]. However, it is becoming increasingly paramount that this proliferation of context-aware technologies is matched by a suitable and sufficient analysis of context research issues. A sound understanding and appreciation of context can lead to improved usability [4] and is regarded as the key to unlocking the true value of business applications on handheld devices [5]. Dey \& Abowd [6] state that context can 'increase the richness of communication in HumanComputer Interaction (HCI) and make it possible to produce more useful computational services'.

The notion of context has been discussed in many disciplines (e.g. psychology, linguistics, computer science) and has recently triggered more multi-disciplinary interest (partially due to the inconsistencies and ambivalent definitions across different research specializations). While a general and unifying theory or formalisation of context is still in its infancy [7], commonalities do exist, e.g. context is broken down into components/variables and their interactions are addressed. Zetie 
[5] emphasizes the importance of understanding contextual interactions and explains that task analysis is critical for a suitable assessment.

This study involved an investigation of contextual interactions required for route navigation on foot. A preliminary study revealed that existing navigational locationaware applications (e.g. PocketMap City Guide) use a generic representation of contextual information (i.e. same types of information for all users). Our purpose was to illustrate through a series of interviews the heterogeneous nature of users in interaction with their environmental context. The study hypothesis is that people vary individually and collectively in their use of surrounding environmental objects/information to navigate. It is anticipated that this will have an impact on usability design requirements for navigational context -aware applications.

\section{Methodology}

After a pilot study of four participants, the main interview study was finalised and consisted of 24 participants (12 males and 12 females). Four participants ( 2 males and 2 females) fell into each of the six age categories: 18 or under, 19-25, 26-35, 36$45,46-65$, and 66 or over. All participants were resident in Greater Glasgow and their professions ranged from school pupils to a retired lecturer. The interview study was comprised of three parts:

1. Pre-interview questionnaire: Information on participants' personal details, familiarity with Glasgow centre and knowledge of context -aware computing.

2. Interview: The main interview consisted of four destinations that participants had to describe how to reach on foot. Two destinations were described verbally and two in writing.

3. Post-interview questionnaire: Information on participants' opinions on the importance of different types of contextual information for route navigation, design issues relating to usability and their mobile needs/requirements.

For part 2, well known destinations and a suitable starting point were chosen. The selection criteria were that each destination must be a similar distance from the starting point (approx. 10 minutes), and in different directions. The order by which participants were presented with each destination was randomised and the verbal/written order was alternated (with an equal balance of those who had to write first with those who had to verbalise first). Each interview was recorded in full.

In line with techniques used for verbal protocol analysis [8], participants' descriptions from part 2 involved a subjective categorization of different types of contextual information into nine categories: directional (e.g. left/right, north/south), structural (e.g. road, monument, church), textual-structural based (e.g. Border's bookshop, Greave Sports), textual-area/street based (e.g. Sauchiehall St., George Sq.), environmental (e.g. hill, river, tree), numerical (e.g. first, second, 100m), descriptive (e.g. steep, tall), temporal/distance based (e.g. walk until you reach... or just before you get to...), and sensory (smell/hearing/ touch) (e.g. sound of go-kart engines while passing ScotKart Centre or smelling hops near a brewery). Accumulated scores were calculated each time a participant mentioned/wrote a word/phrase relating to one of the listed contextual categories. 


\section{Results}

The results of the interview study are represented in figures 1-6.
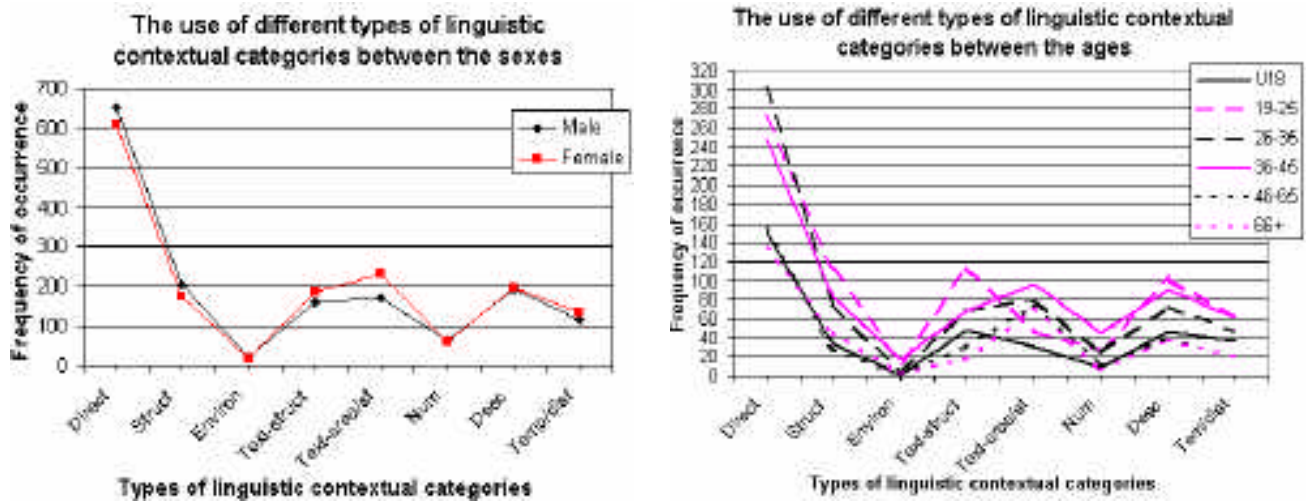

Fig. 1. \& Fig. 2. Use of contextual information between the sexes and ages

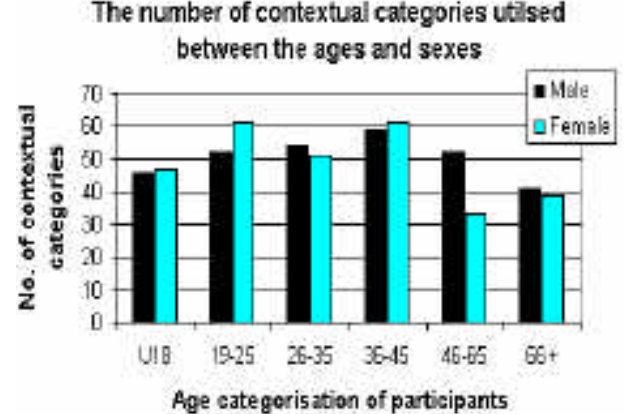

Fig. 3. Number of contextual categories in total for each group by age and sex

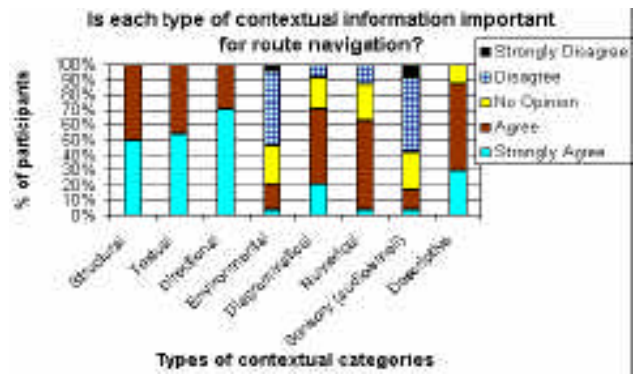

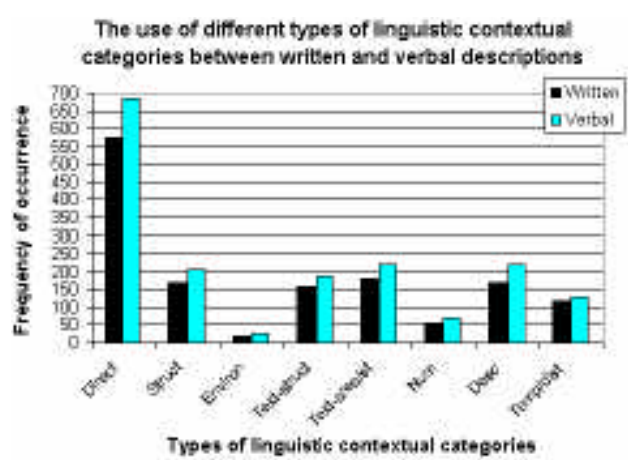

Fig. 4. The use of contextual information for written and verbal descriptions

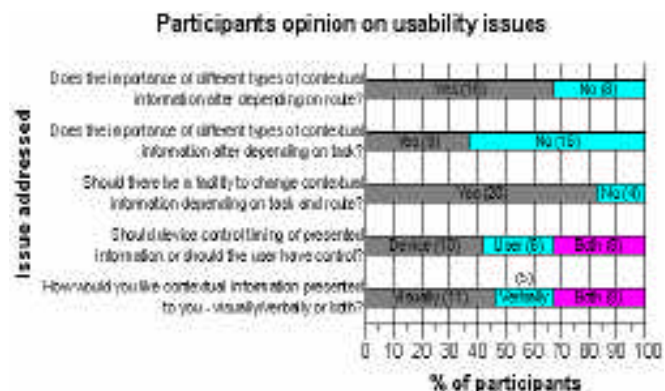

Fig. 5. Participants' opinions on the importance Fig. 6. Participants' opinions on usability issues of contextual information 
The key findings from graphs 1-6 are:

?? There is little difference between sexes in use of different types of linguistic contextual categories (Figure 1). Though, female participants used marginally more textual-structural and textual-area/street based information, whereas males used slightly more directional and structural information.

?? The age groups 26-35, 36-45, 46-65 and 66+ all used more textual-area/street based information in comparison to textual-structural information (Figure 2). However, this trend was reversed for age groups under 18 and 19-25.

?? Participants over 18 but under 45 used significantly more contextual information than those over 45 (Figure 2) (1\% sig-level on 2-tail independent t-test, $\mathrm{t}=7.4 \mathrm{df}$ =38). This is further illustrated in Figure 3 where the under-18s, 46-65s and over $66 \mathrm{~s}$ used less contextual categories than the others. Under-18s were not significantly different from over $45 \mathrm{~s}$.

?? More words/phrases from each type of contextual category were used for verbal descriptions in comparison to written descriptions (Figure 4) (1\% sig-level, 2-tailed correlated t-test, $\mathrm{t}=4.01, \mathrm{df}=23$ ).

?? Most participants either agreed or strongly agreed that structural (100\%), textual $(100 \%)$, directional $(100 \%)$, diagrammatic $(71 \%)$, numerical $(63 \%)$ and descriptive $(88 \%)$ are important for route navigation (Figure 5). Whereas most participants disagreed or strongly disagreed that environmental (54\%) and sensory (58\%) contextual information were important.

?? Most participants $(83 \%)$ would desire a facility to change the type of presented contextual information (Figure 6). Most participants (42\%) would prefer the device to dictate when new contextual information should be presented (Figure 6). Also, most participants (46\%) desire contextual information to be presented visually.

\section{Discussion \& Conclusions}

The results support the original hypothesis that people will vary individually and collectively in their use of contextual information to navigate/orientate. Figure 2 illustrates significant differences between ages, the mo st noticeable being a greater use of textual-structural information than textual-area/street based information by the younger age groups (under 25s). Although the explanation for this trend is outwith the scope of the study, possible reasons could be differences in social behaviour, or that with time, people's geographic knowledge increases enabling a more concise description using mainly street names (textualstreet based information).

The results demonstrate how each participant's contextual descriptions of the environment are unique, and so indicates support for allowing the user to tailor presented information for his/her own needs; a view supported by $83 \%$ of participants (Figure 5). Structural, textual and directional were viewed to be important for route navigation by all participants, but there were differences of opinion for other categories. While environmental and sensory information were rated low, there may be situations were this would change (e.g. we are currently repeating the study for vis ually impaired people).

In line with Dey [4], the results also emphasise the need to understand contextual interactions in order to maximise usability. There were differences in information 
presentation styles (verbal vs. visual) and clear preferences for control over contextual information. The main usability implication/issue is therefore that the design of the application must allow an element of user control in order to present contextual information that is appropriate to a user's task and situation. For example, some participants described of scenarios where speech output would be better for reaching a destination promptly (minimising visual checks) involving concise information (i.e. directional, textual-area/street based and/or textual-structural based information). Whereas, visual presentation involving additional information (e.g. descriptive, numerical, etc) may be preferred (or used in conjunction with speech output) when touring a city for the first time in order to provide a greater spatial orientation and awareness of surrounding environmental features/landmarks. Lastly, another usability implication, based on the results from Figure 4, may be that more contextual information needs to be provided for speech output than for visual presentation.

It is anticipated that the study results will facilitate the design of future navigational context-aware applications. By understanding the dynamic nature of a user's contextual interactions, application designers can better determine which behaviours to support and how to support them. The next stage of our work involves designing a multi-category mobile navigation tool for controlled user experiments while developing a model of contextual interactions encompassing a multidisciplinary appreciation.

\section{References}

1. Chen, G. \& Kotz, D. A Survey of Context-Aware Mobile Computing Research. Technical Report. TR2000-381. Department of Computer Science, Dartmouth College. (2000).

2. Cheverst, K., Davies, N., Mitchell, K., Friday, A. \& Efstratiou. Developing a Context Aware Electronic Tourist Guide: Some Issues and Experiences. Proc CHI 2000. (April 2000), $17-24$.

3. Asthana, A., Cravatts, M. \& Krzyanowski, P. An indoor wireless system for personalized shopping assistance. Proc IEEE Workshop on Mobile Computing Systems and Applications, Santa Cruz, California, (December 1994), 69-74.

4. Dey, A.K. Supporting the Construction of Context-Aware Applications. Dagstuhl seminar on Ubiquitous Computing, (September 2001).

5. Zetie, C. Unwired Express website: Market Overview - The Emerging Context-Aware Software Market. (2002) http://www.unwiredexpress.com

6. Dey, A.K. \& Abowd, G.D. Towards a Better Understanding of Context and ContextAwareness. Proc CHI 2000 Workshop on The What, Who, Where, When, and How of Context-Awareness. The Hague, Netherlands, (April 2000).

7. Benerecetti, M., Bouquet, P. \& Ghidini, C. On the Dimensions of Context Dependence: Partiality, Approximation, and Perspective. Modeling and Using Context: Proc. 3 International Conference, CONTEXT 2001, Dundee, Scotland (July 2001) 59-72.

8. Bainbridge, L. Verbal Protocol Analysis. In Wilson, J.R. \& Corlett, E.N. (Eds.), Evaluation of Human Work: A practical Ergonomics Methodology. London: Taylor and Francis, (1991), 161-179. 